\title{
Voltage Noise Measurement of a REBCO Superconducting Coil During Electromagnetic Vibration Tests on a Ground Coil
}

\author{
Katsutoshi MIZUNO \\ Masafumi OGATA \\ Cryogenic systems Laboratory, Maglev Systems Technology Division \\ Minoru TANAKA \\ Electromagnetic systems Laboratory, Maglev Systems Technology Division
}

\begin{abstract}
In electromagnetic vibration tests, a ground coil is excited under the strong magnetic field of a superconducting magnet. Since the vibration mechanisms in the test are the same as for actual maglev (magnetic levitation) running conditions, electromagnetic vibration tests are therefore an important durability test for ground coils. We carried out electromagnetic vibration tests with a superconducting magnet with REBCO wire, which is a high-temperature material. This paper also proposes a novel monitoring and protection method for REBCO magnets.
\end{abstract}

Key words: electromagnetic vibration test, REBCO, ground coil, magnet protection

\section{Introduction}

In the superconducting maglev systems, propulsion, levitation, and guidance force are generated by ground coils and on-board superconducting magnets. The superconducting magnets are DC excited, and the passing vehicle causes induction current in the ground coils. The interaction between the DC field of the magnets and the induced current in the ground coil generates levitation, and guidance force. Propulsion force is generated by $\mathrm{AC}$ excitation of the ground coils.

A huge number of ground coils are lined up along the guide way. As the ground coils are a key component of maglev systems and must be highly reliable, tests to verify their durability are very important. One of these ground coil durability tests is the electromagnetic vibration test. In this test an AC energized ground coil is placed under a magnetic field generated by a super conducting magnet, and an electromagnetic force acting on the ground coil is generated to obtain a vibration force simulating the running of a vehicle. RTRI has been conducting this type of electromagnetic vibration test for a long time [1]. However, a world-wide helium shortage, is threatening to make the operation of low temperature superconducting (LTS) magnets difficult in the future. As such, RTRI has also been developing high temperature superconducting (HTS) magnets, which use Rare-Earth Barium Copper Oxide (REBCO) coated conductors, for application to maglev or fly wheel energy storage systems [2]. Based on the techniques and the know-how obtained through these developments, we built a REBCO magnet for electromagnetic vibration tests. REBCO has a high critical temperature, therefore liquid helium is not needed to operate REBCO magnets. Furthermore, its short cooling time makes it possible to speed-up experiment cycles and reduce the cost of experiments.

The time required to conduct electromagnetic vibration tests using REBCO magnets is something that needs to be considered. Ten days or more are needed for a lifetime evaluation of a ground coil even in an accelerated test. As the REBCO magnet remains in a state of excitation day-and-night without an operator, a detection and protection system against unexpected situations is indispensable. Because of the difference in operating temperature and cooling system between HTS and LTS, the transition from superconducting state to normal state of HTS magnets is totally different from that of LTS magnets. The detection and protection system for an HTS mag- net must therefore be developed from scratch.

The REBCO magnet in this research was designed especially for electromagnetic vibration tests. However, the magnet's design, manufacturing method, detection and protection system can be used for other REBCO applications. This paper describes the electromagnetic vibration test of a ground coil using the REBCO magnet and the detection and protection method for the REBCO magnet.

\section{Electromagnetic vibration test}

Since the development of the REBCO magnet for the electromagnetic vibration test has been introduced in our previous paper [3], we mention only important features here: the configuration of the magnet and the specifications of the outer vessel. A shorter electromagnetic vibration test was carried out to confirm that the induced electromagnetic force was equivalent to that found in an actual maglev system. The magnet conditions were also checked during the vibration test.

\subsection{Specifications of the REBCO magnet}

The electromagnetic force depends on the magnetic field by the superconducting magnet. Therefore, it is ideal that the dimensions and the magnetomotive force of the superconducting coil are the same as those in the on-board magnet for the maglev. From the viewpoint of heat load to the magnet, the material and design of the outer vessel are also important. This is because the eddy current heating in the REBCO coil is strongly affected by the shielding effect on the outer vessel, which cancels the AC magnetic field with the eddy current in the conducting body. The shielding effect is required to be as strong as that of the on-board magnet. In other words, the REBCO magnet for the electromagnetic vibration test resembles the onboard magnet. However, the number of poles can be different. While an onboard magnet has 4 poles, a one-pole or two-pole magnet can be used for vibration tests. There are different types of ground coil for different functions. For instance, a levitation and guidance coil can be vibrated by a one pole magnet, while a propulsion coil requires a two-pole magnet. The REBCO magnet for this research was a two-pole design with a preinstalled REBCO coil. We planned to conduct vibration tests on levitation and guidance coils in 
parallel with the manufacturing of the second REBCO coil. The outer vessel is totally different from that of the LTS on-board magnet. The outer vessel of the LTS magnet is made of pure aluminum plate on the ground coil side and aluminum alloy for the other sides. The outer vessel of this REBCO magnet was made of 6061 aluminum alloy. Although the shielding effect was slightly reduced, the eddy current heating only accounts for small portion of the total heat load. Confirmation was obtained that it could be ignored in simulations and experiments [4]. Another feature is the manufacturing method which applies machining instead of welding. As shown in

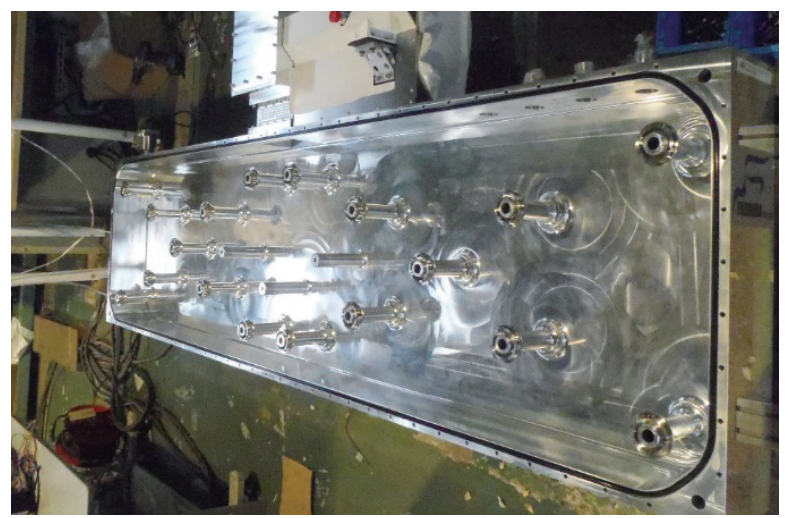

Fig. 1 Outer vessel of the REBCO magnet for electromagnetic vibration test

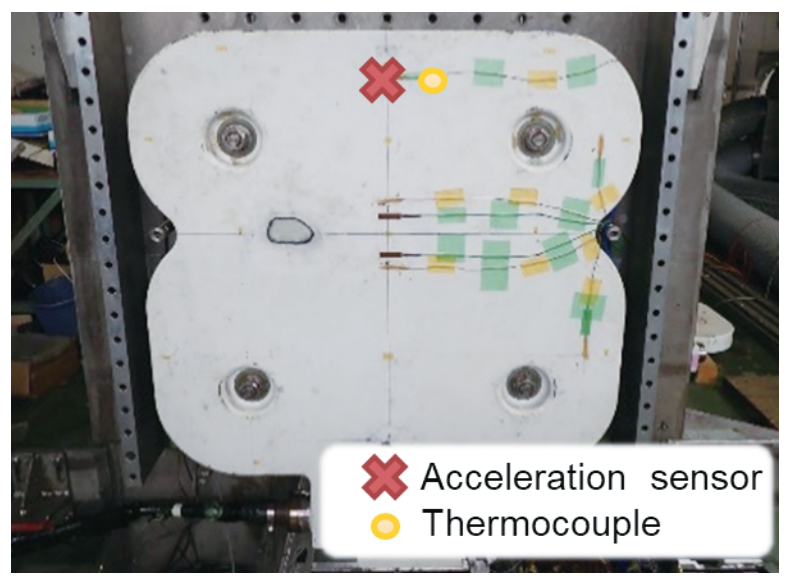

Fig. 2 PLG coil and sensor position

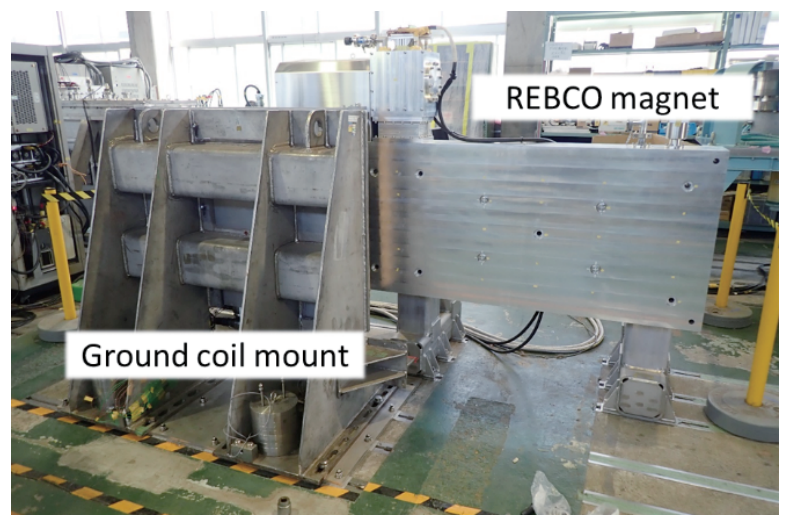

Fig. 3 Constitution of the REBCO magnet and the ground coil for the electromagnetic vibration test
Fig. 1, pillars are integrated into the outer vessel and there are fewer parts.

In comparison to LTS magnets, REBCO magnets are expensive because of the cost of the conductors. Therefore, in order to make REBCO magnets viable, it is essential to further reduce the cost of the component parts and increase their added value. Even though cryogen tanks and pipes for liquid nitrogen and liquid helium are not necessary, it is not sufficient in terms of cost reduction. Therefore, the second REBCO coil design should differ from the first REBCO coil. The total amount of the REBCO coated conductor should be reduced and the coil structure should be simplified.

\subsection{Experimental conditions}

We chose a PLG (combined Propulsion, Levitation and Guidance system) coil [5] for the electromagnetic vibration test (see Fig. 2), since it can be tested with a one-pole REBCO magnet. Previous PLG coil vibration tests were carried out using an LTS magnet. The data obtained in the previous vibration tests using the LTS magnet was helpful to understand the experimental data with the REBCO magnet. The shape of the PLG coil is a vertical asymmetric figure-of-eight. Based on the electromagnetic principle of the shape, it can generate levitation force, guidance force and propulsion force by controlling the excitation current.

In the vibration test, positional relation of the REBCO magnet and the PLG coil were adjusted to that in the maglev system to reproduce the actual electromagnetic force (see Fig. 3). The REBCO coil was DC excited with the magnetomotive force of $700 \mathrm{kA}$. The excitation condition of the PLG coil was levitation mode. The upper loop coil is excited with the combination of DC 500 A and AC 500 A $100 \mathrm{~Hz}$, and the lower loop coil was excited with the combination of DC $555 \mathrm{~A}$ and AC $555 \mathrm{~A} 100 \mathrm{~Hz}$. The PLG coil was excited intermittently: 5 second for excitation and 300 second for cooling down. This is because ground coils are only excited for a short time while the maglev vehicle passes through, and are not designed for continuous excitation. The temperature rise of the PLG coil due to the joule heating was reduced by a blower attached on the top of the ground coil mount. In the experiment, the PLG coil was excited and cooled down in 13 cycles, and the temperature rise of the PLG coil and the REBCO and the vibration waveform were measured.

\subsection{Experimental result}

Figure 4 (a) shows the temperature of the REBCO magnet during the vibration test. Since multiple temperature sensors were attached on the REBCO coil, the average coil temperature and the cryocooler temperature are shown here. Strictly speaking, each temperature measurement of the REBCO coil and cryocooler increased slightly. However, we already confirmed that this temperature rise was from the magnet excitation: the excitation current causes the joule heating at the current leads. The same temperature rise was observed during magnet excitation even when the ground coil was not excited. While the upper limit temperature of the magnet excitation was $39 \mathrm{~K}$, the coil temperature was $32 \mathrm{~K}$. This sufficient temperature margin indicates that long term operation is possible.

A thermocouple was inserted into the resin layer near the conductor of the PLG coil, at the top of the upper loop coil, as shown in Fig. 2. As shown in Fig. 4 (b), the PLG coil temperature changed in a triangular wave shape every energization ( $5 \mathrm{~s})$ and energization suspension (300 s), and increased with time on average. After that, the temperature rise became gradual, and the coil temperature was almost stable in the range from $44^{\circ} \mathrm{C}$ to $47^{\circ} \mathrm{C}$ finally. Meanwhile, 
periodic noise was observed in the REBCO coil temperature. This was caused by the fluctuating magnetic field of the PLG coil. Therefore, it was synchronized with the excitation timing. The noise level was small enough and did not affect the monitoring of the REBCO magnet.

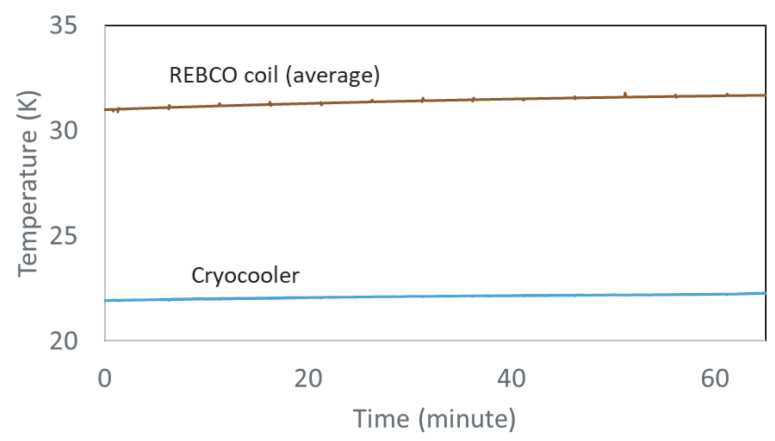

(a) REBCO magnet (Absolute temperature)

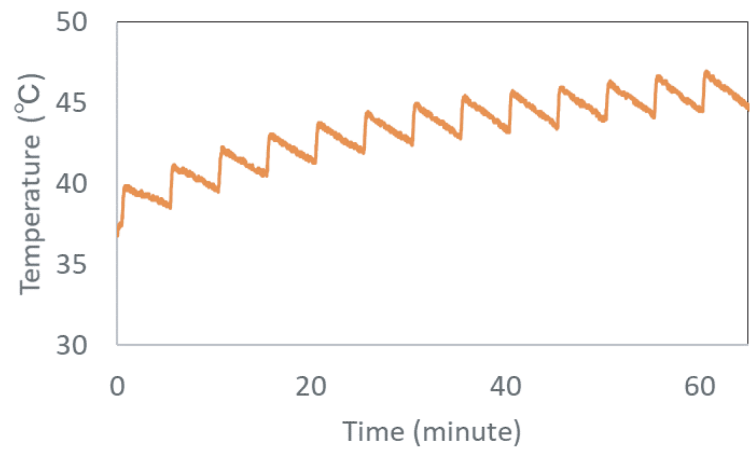

(b) PLG coil (near the conductor of the upper loop coil, Celsius temperature)

Fig. 4 Temperature during the electromagnetic vibration test

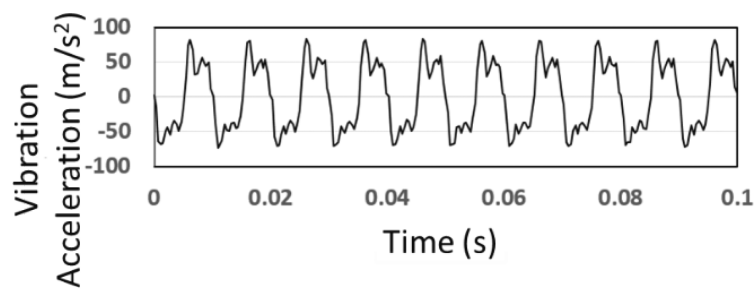

(a) Under the magnetic field generated by the REBCO magnet

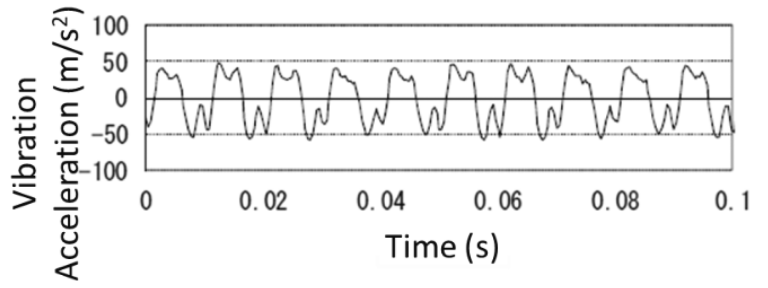

(b) Under the magnetic field generated by a LTS magnet

\section{Fig. 5 Vibration acceleration during the electromagnetic} vibration test
Figure 5 shows the vibration waveform of the PLG coil. The position of the acceleration sensor is next to the thermocouple (see Fig. 2). The vibration amplitude is approx. $50 \mathrm{~m} / \mathrm{s}^{2}$, which is almost the same as that in the vibration test with an LTS magnet. These results indicate that adequate vibration force is given to the ground coil by a REBCO magnet. Incidentally, sharp peaks of approximately $100 \mathrm{~m} / \mathrm{s}^{2}$ appear only in Fig. 5 (a). The difference of the waveform depends on the fixing condition of the PLG coil, especially current leads.

\section{Voltage noise in the REBCO coil from the ground coil}

As mentioned in chapter 2, the REBCO magnet can generate the necessary electromagnetic force for the electromagnetic vibration test. However, the vibration test continues for several weeks in some cases, and there is a possibility of blackout or malfunction of the apparatus. The worst case is thermal runaway of the REBCO coil. If a malfunction of the cooling system is not detected and the excitation of the REBCO coil continues, the coil may burn due to the normal transition. Therefore, an automated early detection of the malfunctions and shut down of the excitation power supply are indispensable for long-term REBCO magnet operation. The normal transition detection based on coil voltage monitoring was proposed in our previous study [6]. Since the voltage of the normal transition is infinitesimal in the early stages, voltage noise reduction method is essential. Noise due to power supply can be reduced by using the difference in pancake coil voltages. The pancake coil is a designation of a flattened coil, and a large REBCO coil usually consists of stacked pancake coils. Therefore, the total REBCO coil voltage is the sum of the voltages of the stacked pancake coils. However, in the electromagnetic vibration test, noise due to the fluctuating magnetic field of the ground coil must be removed.

\subsection{Voltage noise measurement of the REBCO coil}

Voltage noises in the REBCO coil were measured using the experimental configuration for the vibration test in chapter 2. Since this measurement was focused on the noise due to the PLG coil excitation, the REBCO coil was just cooled down without excitation. Although most of the fluctuating magnetic field is shielded by the outer vessel, some magnetic field reaches the REBCO coil and induces voltage. As shown in Fig. 6, the REBCO coil consists of 8

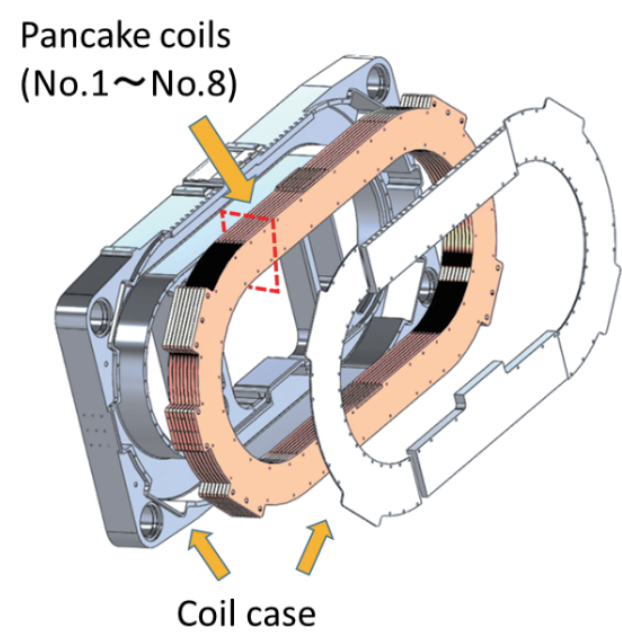

Fig. 6 Configuration of the REBCO coil 


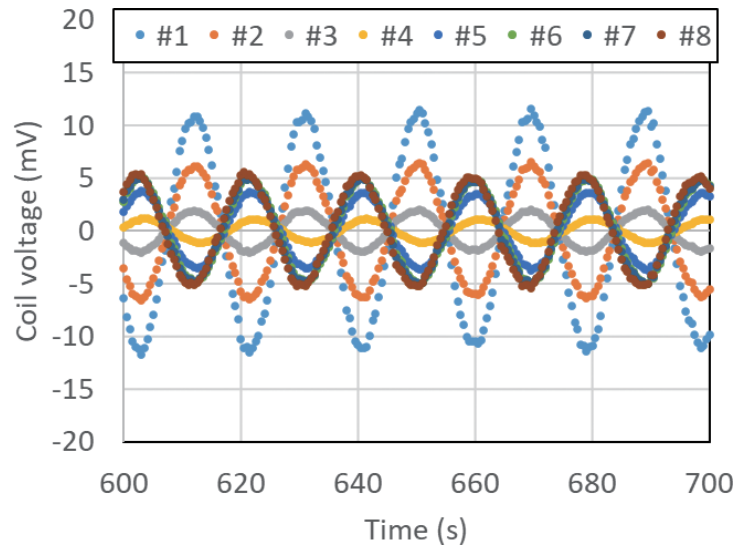

Fig. 7 Voltage noise of the REBCO coil during PLG coil excitation (200 A, $40 \mathrm{~Hz}$, levitation mode)

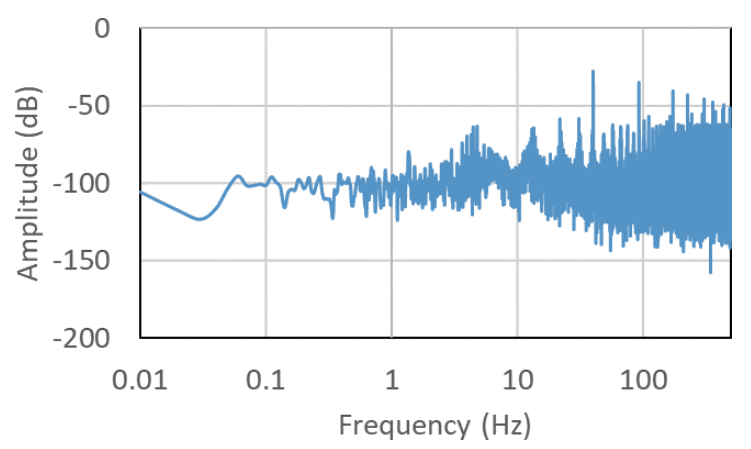

Fig. 8 Frequency response of the \#1 pancake coil during PLG coil excitation

pancake coils (No. 1 No. 8). The voltage noises for each pancake coil are shown in Fig. 7. Although it seems as if the noises have periodic patterns about $20 \mathrm{~s}$, this is because of the alias due to the low sampling rate. As shown in Fig. 8 which is the result of FFT using a high-speed logger, the frequency band is wide. It is noted that a sharp peak at $40 \mathrm{~Hz}$ is the frequency of the excitation current of the PLG coil.

Figure 9 (a) shows the differences of the voltages of two pancakes coils in symmetric positions. Although this difference method is effective in reduction of the voltage noise due to power supply, the voltage noise from the PLG coil excitation is enhanced. The maximum noise amplitude is $16 \mathrm{mV}$. In a thermal runaway test using a small REBCO coil [6], detection level of $0.5 \mathrm{mV}$ was required to prevent the burn-out of coil. The normal voltage of $0.5 \mathrm{mV}$ is easily buried in the noise, so that detection is impossible. Figure 9 (b) and (c) are also the differences in pancake coil voltages under different excitation conditions of the PLG coil. The wave shapes of the voltage differences (\#1-\#8,\#2-\#7, \#3-\#6 and \#4-\#5) have similar forms. Moreover, the similarity ratios are constant regardless of the excitation condition of the PLG coil. Thereby, the noise can be canceled by (1).

$$
\begin{aligned}
& a=V_{\# 1-\# 8}-1.47 V_{\# 2-\# 7} \\
& b=V_{\# 2-\# 7}-1.67 V_{\# 3-\# 6} \\
& c=V_{\# 3-\# 6}-2.63 V_{\# 4-\# 5}
\end{aligned}
$$

Figure 10 shows the noise-canceled coil voltages. It was found that the noise levels were reduced to one twentieth of those in Fig. 9. The noise level was about $3 \mathrm{mV}$ in some conditions. However, since high-speed detection is unnecessary for the coil protection, low pass

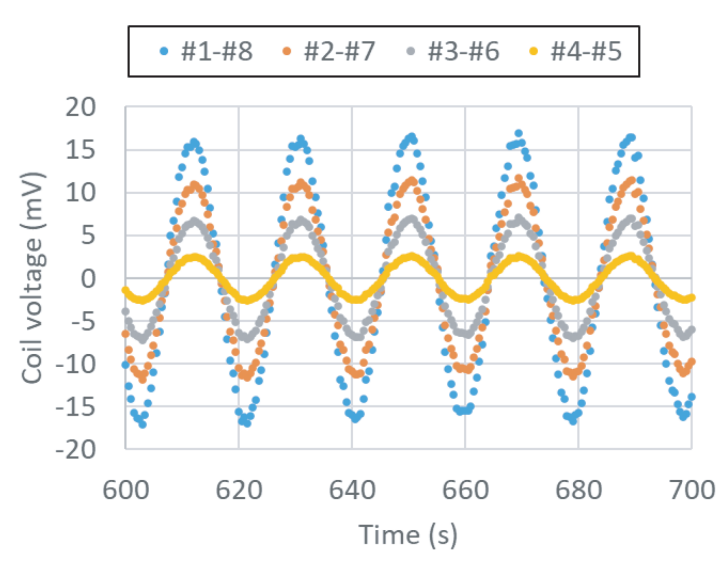

(a) $200 \mathrm{~A}, 40 \mathrm{~Hz}$, levitation mode

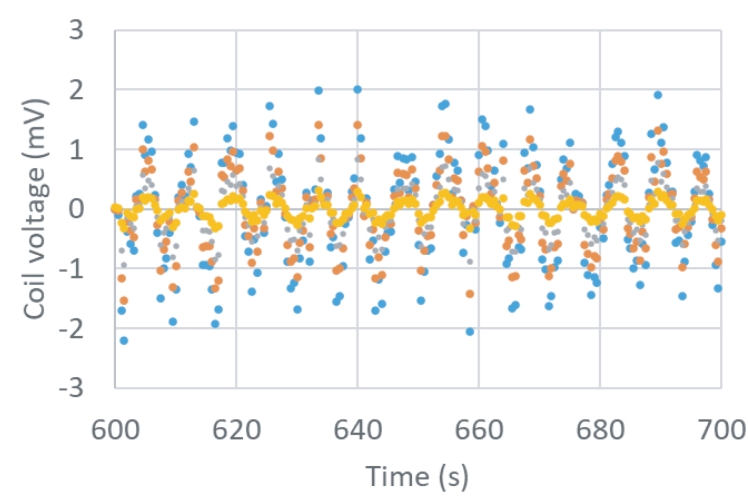

(b) $200 \mathrm{~A}, 120 \mathrm{~Hz}$, levitation mode

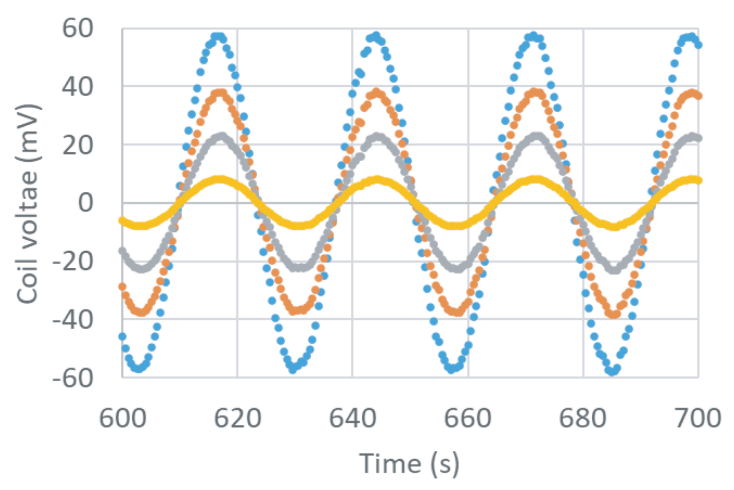

(c) $200 \mathrm{~A}, 40 \mathrm{~Hz}$, propulsion mode

\section{Fig. 9 Voltage noise of the REBCO coil in different exci- tation condition of the PLG coil (before the noise canceling)}

filter can be used. The combination of the noise reduction methods and the low pass filter improved measurement accuracy.

The experimental conditions were different from the electromagnetic vibration test or the actual maglev operation because of the limitations of the experimental apparatus. The voltage noise measurements were carried out under more stringent conditions. In the vibration test, a pure aluminum plate was placed between the REBCO magnet and a ground coil. Although the excitation current was high (about $500 \mathrm{~A}$ ), almost all the fluctuating magnetic field was shielded by the aluminum plate. Meanwhile, it should be noted that the frequency of the magnetic field is $309 \mathrm{~Hz}$ in maglev opera- 


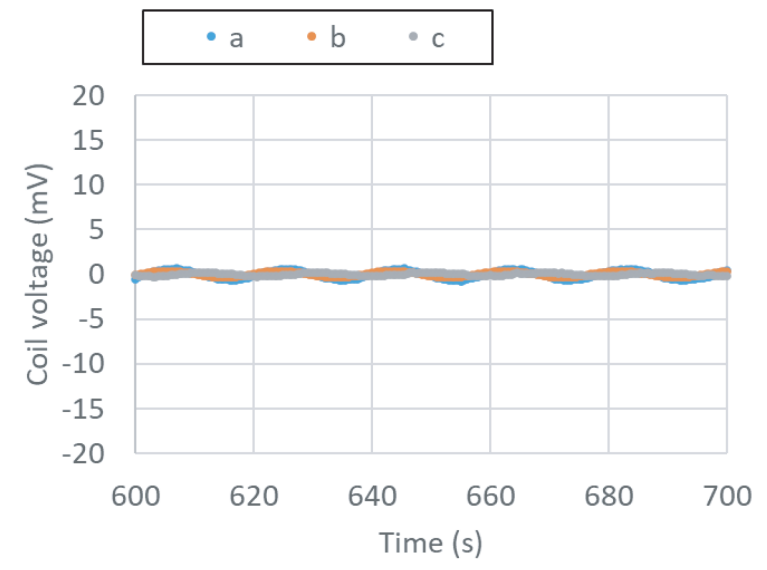

(a) $200 \mathrm{~A}, 40 \mathrm{~Hz}$, levitation mode

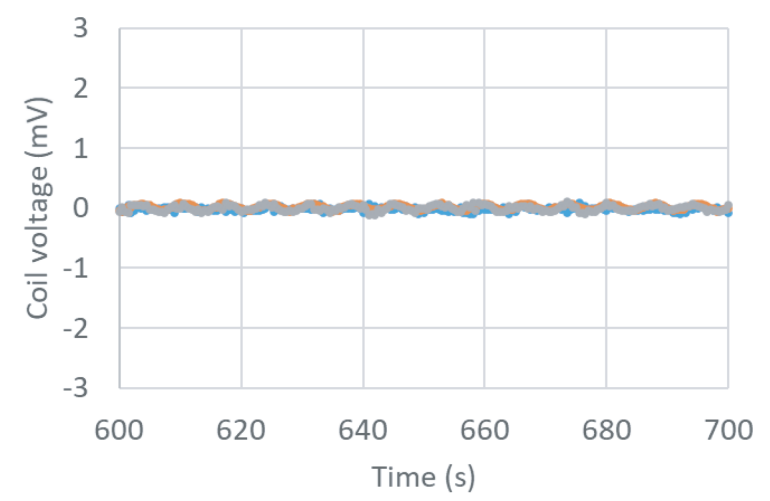

(b) $200 \mathrm{~A}, 120 \mathrm{~Hz}$, levitation mode

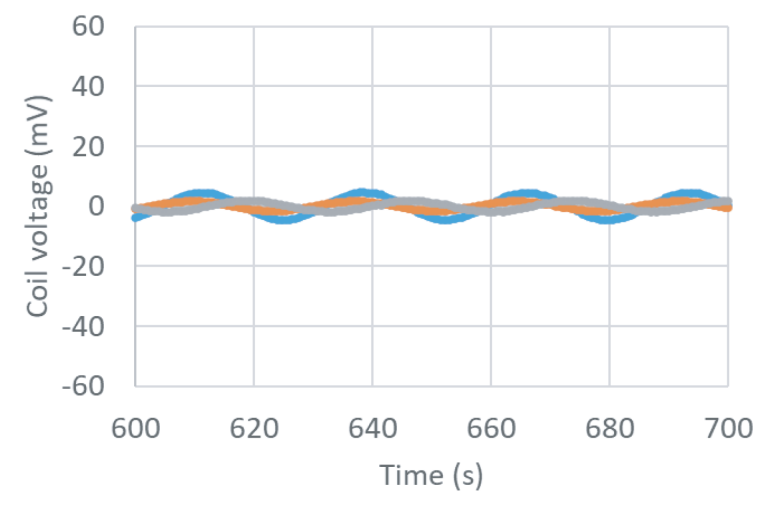

(c) $200 \mathrm{~A}, 40 \mathrm{~Hz}$, propulsion mode

Fig. 10 Voltage noise of the REBCO coil in different excitation condition of the PLG coil (after the noise canceling)

tions. As the shielding effect increases at high frequency, the fluctuating magnetic field which reaches to the REBCO coil is relatively small.

\subsection{Noise canceling mechanism}

These results of the noise voltage measurements of the REBCO coil revealed that the noise patterns on the pancake coils were homothetic regardless of the excitation condition of the ground coil. This fact can be explained as follows: the REBCO coil in this re-

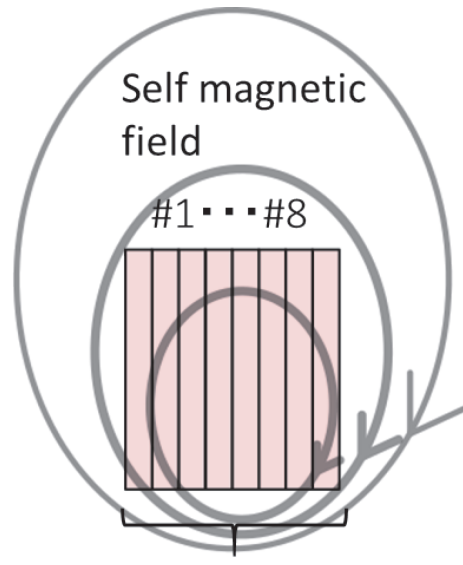

Pancake coils \#1 \#8

(a) Self-magnetic field by the excitation of the REBCO coil

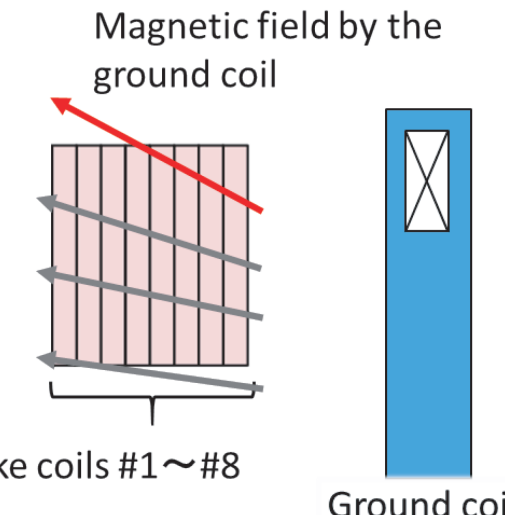

(b)Magnetic field by the excitation of ground coil

Fig. 11 Schematic drawing of the magnetic flux in the cross section of the REBCO coil

search consisted of 8 pancake coils and a coil case (see Fig. 6). The pancake coils were stacked and connected in series. A schematic drawing of the magnetic flux in the cross section (red broken line area in Fig. 6) is shown in Fig. 11. The magnetic flux by the REBCO coil itself is bilaterally symmetric (see Fig. 11 (a)). Therefore, pancake coils in bilaterally symmetric position have not only the same mutual inductance but also the same magnetic flux distribution. For that reason, the difference of the pancake coil voltages cancels the noise due to the excitation power supply and shielding current [6]. The shielding current is a loop current inside the superconducting layer of the REBCO coated conductor caused by fluctuating magnetic field. As the normal transition is a local phenomenon, this difference method dose not remove the voltage due to the transition.

Meanwhile, the magnetic flux from the ground coil is asymmetric as shown in Fig. 11 (b). Here, the difference between the two pancake coil voltages (e.g., \#1 and \#8) means the induced voltage from the flux goes through only one of the pancake coils. The reason is that the induced voltage from flux which goes through both is canceled. In Fig. 11 (b), only the flux shown in red arrow remains. If the magnetic flux density is assumed to be uniform in this area, the induced voltage is proportional to on the distance of the pancake coils. The ratio of the physical distances of the pancake coils are 
given by (2).

$$
\frac{L_{\# 1-\# 8}}{L_{\# 2-\# 7}}=1.4, \frac{L_{\# 2-\# 7}}{L_{\# 3-\# 6}}=1.67, \frac{L_{\# 3-\# 6}}{L_{\# 4-\# 5}}=3
$$

Where, "L" means the physical distance of the pancake coils. These ratios are close to the homothetic ratios of the noise patterns (1). The slight differences are considered due to the shielding effect in the heat transfer members between the pancake coils.

\section{Conclusions}

This paper described the development of a REBCO magnet for electromagnetic vibration tests on ground coils. The REBCO magnet does not require cryogen such as liquid helium or liquid nitrogen and increases the speed of the vibration test cycle. Although the REBCO magnets only currently have a one-pole configuration, a PLG coil was vibrated by using the magnet. The magnet remained in a stable state during the vibration test, and adequate vibration force worked on the PLG coil.

In addition, we developed a detection and protection method required for long-term vibration tests. In order to detect normal transition voltage at an early stage, noise reduction methods in the coil voltage are essential. Minute voltage due to the normal transition can be obtained by the combination use of following methods.

(1) Difference in pancake coil voltages in symmetric positions for the cancellation of the noise due to the excitation power supply and shielding current

(2) Noise reduction using similarity ratio of noise patterns of the ground coil

(3) Improvement of measurement accuracy using a low pass filter

We are planning to conduct long-term electromagnetic vibration tests of the levitation and guidance coils. In addition, we aim to produce a second REBCO coil.

\section{References}

[1] Tanaka M., Aiba M., Suzuki M., "Development of Electromagnetic Vibration Test Apparatus for Ground Coils Applied to Maglev System," Quarterly Report of RTRI, Vol. 48, No. 2, pp. 110-114, 2007.

[2] Miyazaki, Y. et al., "Development of a Superconducting Magnetic Bearing Capable of Supporting Large Loads in a Flywheel Energy Storage System for Railway Application," Quarterly Report of RTRI, Vol. 61, No. 1, pp. 54-59, 2020.

[3] Mizuno, K., Sugino M., Tanaka, M., Ogata, M., "Development of a Real-scale REBCO Coil for the Demonstration of a Magnetomotive Force of 700 kA," Quarterly Report of RTRI, Vol. 58, No. 4, pp. 318-322, 2017.

[4] Mizuno, K., Tanaka, M., Ogata, M., "Evaluation of Eddy Current Heating in a REBCO Magnet Due to the Magnetic Field of Ground Coils for the Maglev," Superconductor Science and Technology, Vol. 33, No. 7, 074009, 2020.

[5] Murai T., "Characteristics of LSM Combined Propulsion, Levitation and Guidance," T. IEE Japan, Vol. 114, No. 7/8, pp. 746752, 1994 (in Japanese).

[6] Mizuno, K., Tanaka, M., Ogata, M., "Monitoring and Protection Method of a REBCO Magnet Designed for Electromagnetic Vibration Tests," Quarterly Report of RTRI, Vol. 61, No. 1, pp. 60-65, 2020.

\section{Authors}

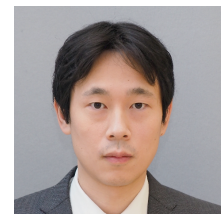

Katsutoshi MIZUNO, Dr. Eng.

Assistant Senior Researcher, Cryogenic

Systems Laboratory, Maglev Systems

Technology Division

Research Areas: Superconducting Technology

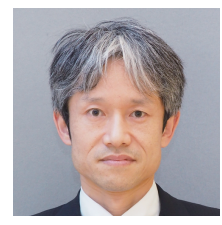

Minoru TANAKA, Dr. Eng.

Senior Chief Researcher, Head of

Electromagnetic Systems Laboratory, Maglev

Systems Technology Division

Research Areas: Electrical Engineering,

Superconducting Technology

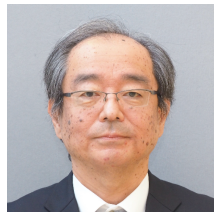

Masafumi OGATA, Dr. Eng.

Senior Chief Researcher, Head of Cryogenic

Systems Laboratory, Maglev Systems

Technology Division

Research Areas: Superconducting Technology 